\section{O Método Mãe Canguru em hospitais públicos do Estado de São Paulo, Brasil: uma análise do processo de implantação}

\author{
Kangaroo Mother Care in public hospitals \\ in the State of São Paulo, Brazil: \\ an analysis of the implementation process
}

Ana Júlia Colameo ${ }^{1}$

Marina Ferreira Rea 1,2

\footnotetext{
1 Núcleo de Investigação em Saúde da Mulher e da Criança, Instituto de Saúde, São Paulo, Brasil.

2 Faculdade de Ciências Médicas, Universidade Estadual de Campinas, Campinas, Brasil.

Correspondência A. J. Colameo

Núcleo de Investigação em Saúde da Mulher e da Criança, Instituto de Saúde. Rua Santo Antonio 590, 2o andar, São Paulo, SP 01314-000, Brasil. anajuh@yahoo.com.br
}

\begin{abstract}
This study analyzed the implementation of the Kangaroo Mother method in 28 different São Paulo State public hospitals. Teaching hospitals, Baby-Friendly Hospitals, hospitals with human milk banks, and those with more than 12 trained health professionals showed higher implementation scores. Because of staff resistance to family participation in neonatal care, the Kangaroo Mother method is basically applied in-hospital. Changes in the initial training, including manager awareness-raising and proper financial resource allocation, are necessary for implementation, follow-up, assessment, and feedback.
\end{abstract}

Kangaroo Mother Method; Premature Infant; Health Policy

\section{Introdução}

No Brasil, os recém-nascidos com peso menor que $2.500 \mathrm{~g}$ perfazem $9,2 \%$ dos nascimentos. No Estado de São Paulo, por ano, nascem cerca de 60 mil bebês nessas condições, dos quais, aproximadamente, $8 \%$ morrem no primeiro ano de vida 1 .

Segundo a classificação de Lubchenco 2 , o bebê que nasce com menos que $2.500 \mathrm{~g}$ é chamado de recém-nascido de baixo peso (RNBP), e o com menos de $1.500 \mathrm{~g}$, recém-nascido de muito baixo peso (RNMBP). Lubchenco \& Searls 3 já demonstraram que quanto menor é o peso de nascimento e a idade gestacional maior é a morbimortalidade desses bebês.

Os cuidados convencionais estabelecidos pelo Ministério da Saúde (MS) ${ }^{4}$ nas unidades de neonatologia, ministrados aos recém-nascidos de baixo peso, visam prevenir e tratar os problemas relacionados com a imaturidade dos múltiplos sistemas e com a desnutrição intra-útero. $\mathrm{O}$ tratamento para esses bebês inclui o uso de monitores, ventiladores artificiais, oxigênio, antibióticos, medidas de manutenção do equilíbrio metabólico e volêmico, uso de alimentação parenteral ou enteral, por sonda ou via oral com leite materno ou fórmula infantil, desinfecção dos equipamentos e lavagem adequada das mãos, entre outros. Mesmo que não apresente mais problemas médicos e seja capaz de coordenar a sucção e deglutição, 
o RNBP, nos cuidados convencionais, é mantido em incubadora até que regule, por si mesmo, a temperatura. Em relação à alta hospitalar, o RNBP somente é liberado após atingir um peso em torno de $2.000 \mathrm{~g}$.

Cattaneo et al. 5 referem que a atenção neonatal convencional tem resultado em baixos níveis de mortalidade e que freqüentemente o tratamento nas unidades de terapia intensiva (UTI) neonatal implica em pouco contato entre as mães e os bebês, muitas vezes, por longos períodos, podendo dificultar a amamentação e interferir na formação do vínculo afetivo.

Amamentar precocemente um bebê prematuro é importante para reduzir a perda de peso, aumentar os níveis de glicose no sangue e diminuir a bilirrubina não conjugada no soro 6 . Além disso, o aleitamento materno fortalece os vínculos afetivos 7 e propicia benefícios, em longo prazo, para o desenvolvimento intelectual e neurológico dessas crianças 8 . Bradley \& Casey 9 apontaram que a qualidade da interação entre a mãe e seu filho pré-termo é fundamental para atenuar ou acentuar as dificuldades próprias dessa condição de vulnerabilidade da criança. Strathearn et al. 10 encontraram que a negligência infantil, um subtipo de maltrato, pode levar os RNMBP a uma acentuação do atraso cognitivo, chegando até a algum grau de retardamento mental.

Atualmente, a humanização das unidades neonatais tem sido um objetivo perseguido pela neonatologia 11, que reconhece o desequilíbrio flagrante entre o declínio da mortalidade e o aumento da morbidade dos RNBP.

O Método Mãe Canguru é uma forma de atenção que incentiva e valoriza a presença e a participação da mãe e da família na unidade neonatal. Tem um papel importante para assegurar a saúde do bebê de baixo peso após a alta hospitalar, tanto pela oportunidade de fortalecimento do vínculo afetivo que oferece, como pelas altas taxas de amamentação que proporciona 12.

O Método Mãe Canguru foi idealizado em 1978 na Colômbia, pelos doutores Rey e Martinez, como resposta à superlotação das incubadoras, levando a altas taxas de infecção e morte, além do abandono dos RNBP por ocasião da alta 13 .

Apesar de ter sido contestado inicialmente, algumas experiências exitosas mostraram que o Método Mãe Canguru, ou "pele a pele", em bebês com estabilidade clínica era prazeroso para as mães e relaxante para os bebês, portanto aplicável no mundo desenvolvido 14,15 . Outras publicações, como os estudos de Ludington-Hoe et al. 16,17, mostraram que o método era seguro e que trazia benefícios, tanto biológicos como sociais e psicoafetivos.

No Brasil, os primeiros hospitais que trabalharam com a posição canguru foram os hospitais Guilherme Álvaro, em Santos, e o Instituto Materno Infantil de Pernambuco (IMIP), na Cidade do Recife. A partir de então, alguns hospitais brasileiros começaram a realizar a "Posição Canguru”, isto é, a colocação do recém-nascido em contato pele a pele sobre o peito da mãe 18 .

Em 5 de julho de 2000, foi publicada a Portaria 693/GM que estabeleceu a Norma de Orientação para a Implantação do Método Canguru 19, tornando o Método Mãe Canguru uma política pública.

O MS, o Banco Nacional de Desenvolvimento Econômico e Social e da Fundação ORSA lançaram o Programa de Humanização no Atendimento do Recém-Nascido de Baixo Peso-Método Mãe Canguru e disponibilizaram um treinamento padrão para equipes hospitalares multidisciplinares de cinco profissionais, em centros de capacitação desenvolvidos por esse convênio 18 .

O Método Mãe Canguru consiste em manter o RNBP ligeiramente vestido em contato com o peito de um adulto, alimentá-lo com leite materno e dar alta precocemente, com controles ambulatoriais freqüentes 13 , sendo a incubadora substituída por uma fonte humana de calor direto.

Conforme relatam Carvalho \& Prochnik 18, o Método Mãe Canguru brasileiro tem cinco elementos básicos: (1) alta precoce baseada nas condições clínicas dos RNBP, (2) amamentação exclusiva, (3) posição canguru para prover calor e estímulos, (4) educação e informação das mães, pais e da família nos cuidados dos prematuros e (5) acompanhamento ambulatorial para monitorar o crescimento e o desenvolvimento do bebê.

A Norma de Orientação para a Implantação do Método Canguru dividiu a implantação em três etapas:

- A primeira etapa é relativa à situação do RNBP, que é internado na unidade neonatal para adaptar-se à vida extra-uterina. Nessa fase, a norma determina que haja livre acesso e participação da família nos cuidados com o bebê, estímulo ao aleitamento materno e o contato pele a pele progressivo até a colocação do RNBP em "posição canguru".

- A segunda etapa é relativa à situação do RNBP com condições de ficar em alojamento conjunto contínuo com a mãe onde permanecem em posição canguru pelo maior tempo possível. A mãe exercita a amamentação e fornece os cuidados específicos ao bebê prematuro, ambos apoiados pelos profissionais do hos- 
pital. A norma define critérios de elegibilidade, estadia e alta hospitalar.

- A terceira etapa é a fase domiciliar. O bebê é acompanhado no ambulatório pela equipe responsável pelo método a cada dois ou três dias inicialmente e depois semanalmente até que atinja 2.500 g ou mais, ocasião em que é encaminhado para a rede pública de saúde.

O Estado de São Paulo regulamentou o Método Mãe Canguru em 5 de junho de 2001 como Resolução SS-62 - Normas de Atenção Humanizada ao Recém-nascido de Baixo Peso (Método Canguru) no Estado de São Paulo 20. No final de 2002, em São Paulo, haviam sido treinados 44 hospitais e uma instituição de pesquisa.

\section{Objetivo}

Analisar o processo de implantação do Programa de Humanização no Atendimento do Recém-nascido de Baixo Peso - Método Mãe Canguru em hospitais de alta tecnologia, do Sistema Único de Saúde, com rotinas convencionais de atendimento neonatal, no Estado de São Paulo, a partir do treinamento padrão.

\section{Metodologia}

Trata-se de estudo transversal desenvolvido através do envio de um questionário pelo correio aos 44 hospitais públicos de São Paulo que receberam treinamento oferecido pelo MS até 2002. Para esgotar a obtenção de dados, foram usadas estratégias adicionais como o encaminhamento de um envelope já endereçado e selado para a resposta junto ao questionário, o reenvio do mesmo questionário dois meses depois, contatos telefônicos com disponibilização de um número de fax para as respostas e esclarecimento de dúvidas e, finalmente, o envio do questionário por correio eletrônico. O universo da análise resultou em 28 hospitais. Foi criado um escore a partir dos quesitos definidos pelas normas de implantação. O critério usado na aplicação do escore foi: dois pontos quando cumpria totalmente, um ponto se parcialmente e nenhum se o quesito não era cumprido. O escore geral foi aplicado em todos os hospitais que responderam ter implantado, pelo menos, uma etapa e incluiu os pontos obtidos em todas as etapas. Os escores estratificados (E1, E2, E3), obtidos a partir da separação do cumprimento por etapas, incluíram os hospitais que haviam declarado ter implantado a respectiva etapa e excluíram os que declararam não tê-la implantado.
Esta pesquisa cumpre os princípios éticos contidos na Declaração de Helsinki (1964, reformulada em 1975, 1983, 1989, 1996 e 2000) da World Medical Association, além do atendimento à Resolução 196 do Conselho Nacional de Saúde, de 10 de outubro de 1996 e foi aprovada pelo Comitê de Ética em Pesquisa do Instituto de Saúde.

\section{Resultados}

\section{Perfil dos hospitais}

A Tabela 1 mostra o perfil dos hospitais estudados: quatro são municipais; cinco, estaduais; cinco, filantrópicos; sete, hospitais escola; e sete são organizações sociais de saúde. Do total, 12 são credenciados como "amigos da criança" (HAC), e 11 contam com banco de leite humano (BLH). Dentro desses números, temos que oito são HAC e têm BLH. Do total, 13 hospitais implantaram as três etapas, e quatro hospitais não iniciaram a implantação.

\section{Grau de implantação}

Os 24 hospitais que declararam ter implantado qualquer etapa obtiveram um escore médio de $56,6 \%$. O maior escore obtido foi $75 \%$, e o menor foi de $9,5 \%$.

A Tabela 2 mostra os hospitais agrupados de acordo com o tipo de hospital, o escore geral correspondente e os escores estratificados por etapa. O escore geral foi mais alto nos hospitais escola com $65,5 \%$. As organizações sociais de saúde tiveram escore geral de $60,5 \%$, os hospitais estaduais de $58,6 \%$, os hospitais municipais de 55,3 e os filantrópicos, com as menores médias, de $39,8 \%$. A Tabela 2 mostra que 22 hospitais implantaram o método na UTI neonatal; 19, no alojamento canguru; e 16, no ambulatório de seguimento. O escore estratificado médio foi maior na primeira etapa (E1: $68,1 \%)$, seguido da segunda etapa (E2: $58,8 \%$ ) e, por último, da terceira etapa (E3: 46,6\%).

A Tabela 3 mostra os valores médios dos escores sob a ótica do hospital ser HAC, ou não, e contar com um BLH ou não. Os HAC (10) têm um escore geral médio igual a $66,3 \%$, enquanto que os não HAC (14) têm 50,5\%. O escore geral médio dos hospitais "com $B L H$ " (10) foi de $66,1 \%$, enquanto que os "sem BLH" (14) tiveram 49,7\%. Os HAC e com BLH (7) apresentam um escore médio de $68,2 \%$, enquanto que os que não são HAC e não contam com BLH (11) têm média de 46,4\%.

A Tabela 4 mostra os escores e esses dois fatores separados por etapa de implantação. 
Caracterização dos hospitais. São Paulo, Brasil, 2004.

\begin{tabular}{|c|c|c|c|c|c|c|c|c|c|c|}
\hline \multirow[t]{2}{*}{ Tipo de hospital } & \multicolumn{2}{|c|}{$\begin{array}{l}\text { Número de } \\
\text { hospitais }\end{array}$} & \multicolumn{2}{|c|}{$\begin{array}{l}\text { Amigo da } \\
\text { criança }\end{array}$} & \multicolumn{2}{|c|}{$\begin{array}{c}\text { Banco de leite } \\
\text { humano }\end{array}$} & \multicolumn{2}{|c|}{$\begin{array}{l}\text { Leitos de } \\
\text { UTI neonatal }\end{array}$} & \multicolumn{2}{|c|}{$\begin{array}{c}\text { Leitos Mãe } \\
\text { Canguru }\end{array}$} \\
\hline & $n$ & $\%$ & $\mathrm{n}$ & $\%$ & $\mathrm{n}$ & $\%$ & $\mathrm{n}$ & $\%$ & $\mathrm{n}$ & $\%$ \\
\hline Municipal & 4 & 14,2 & 3 & 25,0 & 1 & 9,1 & 23 & 8,3 & 2 & 2,3 \\
\hline Estadual & 5 & 17,9 & 1 & 8,3 & 3 & 27,3 & 35 & 12,7 & 21 & 24,1 \\
\hline Filantrópico & 5 & 17,9 & 1 & 8,3 & 1 & 9,1 & 37 & 13,4 & 10 & 11,5 \\
\hline Escola & 7 & 25,0 & 6 & 50,0 & 4 & 36,4 & 110 & 39,8 & 30 & 34,5 \\
\hline $\begin{array}{l}\text { Organizações sociais } \\
\text { de saúde }\end{array}$ & 7 & 25,0 & 1 & 8,3 & 2 & 18,1 & 71 & 25,8 & 24 & 27,6 \\
\hline Total & 28 & 100,0 & 12 & 100,0 & 11 & 100,0 & 276 & 100,0 & 87 & 100,0 \\
\hline
\end{tabular}

Tabela 2

Percentual médio de cumprimento das normas de implantação geral (E) e por etapas implantadas (E1, E2, E3), segundo o tipo de hospital. São Paulo, Brasil, 2004.

\begin{tabular}{|c|c|c|c|c|c|c|c|}
\hline \multirow[t]{3}{*}{ Tipo de hospital } & \multirow{3}{*}{$\begin{array}{c}\text { Escore } \\
\text { geral (\%) }\end{array}$} & \multicolumn{6}{|c|}{ Escore médio relativo a cada etapa implantada } \\
\hline & & \multicolumn{2}{|c|}{$\begin{array}{l}\text { UTI ou UCI } \\
\text { neonatal }\end{array}$} & \multicolumn{2}{|c|}{$\begin{array}{l}\text { Alojamento } \\
\text { canguru }\end{array}$} & \multicolumn{2}{|c|}{$\begin{array}{l}\text { Ambulatório } \\
\text { de seguimento }\end{array}$} \\
\hline & & $\mathrm{n}$ & $\mathrm{E} 3(\%)$ & $\mathrm{n}$ & $\mathrm{E} 2(\%)$ & $\mathrm{n}$ & $\mathrm{E} 1(\%)$ \\
\hline Municipal & 55,3 & 2 & 76,6 & 1 & 48,7 & 1 & 41,7 \\
\hline Estadual & 58,6 & 3 & 70,8 & 4 & 62,5 & 4 & 47,9 \\
\hline Filantrópico & 39,8 & 5 & 44,4 & 4 & 53,7 & 2 & 41,7 \\
\hline Escola & 65,5 & 6 & 78,6 & 5 & 66,5 & 4 & 47,9 \\
\hline $\begin{array}{l}\text { Organizações sociais } \\
\text { de saúde }\end{array}$ & 60,5 & 6 & 70,3 & 5 & 64,0 & 5 & 53,6 \\
\hline Total & 56,6 & 22 & 68,1 & 19 & 58,8 & 16 & 46,6 \\
\hline
\end{tabular}

\section{Implantação da primeira etapa}

Foram incluídos 22 hospitais. A média dos escores dessa etapa foi $68,1 \%$. O maior escore da primeira etapa (E1: 78,6\%) foi obtido pelos hospitais escola (Tabela 2). Os hospitais filantrópicos foram os que apresentaram o menor escore dessa etapa (E1: 44,4\%), enquanto que os outros hospitais tiveram suas médias acima de $70 \%$.

Nessa etapa, os hospitais que obtiveram escores médios mais altos foram os HAC e com BLH, com 80,3\%. Quando estudados separadamente, os HAC apresentaram média de $79,4 \%$, e os com BLH, uma média de 76,9\%. Os hospitais sem os dois fatores tiveram média de 53,5\% (Tabela 4).

Os quesitos mais difíceis de cumprir foram: a disponibilidade da equipe para sanar as dúvidas dos pais livremente, fornecer auxílio transporte ou similar para facilitar a vinda dos pais ao hospital, oferecer três ou mais refeições para que a mãe possa passar o dia todo com seu bebê, dispor de um espaço para descansar ou para a formação de grupos ou palestras e estimular a participação do pai nas reuniões com a equipe. A visita da família ao bebê internado na UTI neonatal era permitida; e, em um terço das vezes, a família era acolhida e preparada para as visitas. Nos outros, os critérios adotados eram bastante rígidos e restritivos.

\section{Implantação da segunda etapa}

O número de hospitais incluídos foi 19, com um escore médio de $58,8 \%$ (Tabela 2). O maior escore (E2: 66,5\%) foi obtido pelos hospitais escola. O menor foi obtido pelo hospital municipal avaliado (E2: 48,7\%). 
Os HAC, os que contavam com BLH e os que tinham ambos fatores (HAC+BLH) obtiveram um escore médio de 65,5\%, 65,3\% e 65,7\%, respectivamente. Os hospitais não credenciados como amigos da criança e sem BLH obtiveram um escore de $56,6 \%$ (Tabela 4 ).

Os quesitos mais difíceis de cumprir foram: a exigência de ganhar peso maior que $15 \mathrm{~g} / \mathrm{dia}$ para entrar nessa etapa; a decisão de iniciar o Método Mãe Canguru num consenso entre a equipe, a mãe e os familiares; a freqüência das mamadas diurnas a cada duas horas e noturnas a cada três horas; o peso mínimo de $1.500 \mathrm{~g}$ para a alta (a maioria dos pesos adotados ficou entre $1.750 \mathrm{~g}$ e $2.000 \mathrm{~g}$ ); obter ganho de peso consecutivo nos últimos três dias; não ser alimentado por sonda; o compromisso materno e familiar de realizar o método $24 \mathrm{~h} /$ dia e de obedecer ao cronograma de retorno; a condição de recorrer ao hospital a qualquer momento de urgência.

\section{Implantação da terceira etapa}

Foram incluídos 16 hospitais, e as médias dos escores ficaram abaixo de $50 \%$ na maioria dos hospitais, exceto nas organizações sociais de saúde (E3: 53,3\%), como visto na Tabela 2. Os escores dos hospitais estaduais e hospitais escola foram iguais (E3: 47,9\%). Os escores dos hospitais filantrópicos e do hospital municipal avaliado foram menores (E3: 41,7\%).

Nessa etapa (Tabela 4), os HAC, os com BLH e aqueles com as duas condições obtiveram os mesmos escores (50\%). Os hospitais sem os dois fatores apresentaram o menor escore $(40,3 \%)$.

Os quesitos menos cumpridos foram os relacionados à não observação do primeiro retorno até 48 horas após a alta hospitalar e aos poucos serviços que realizam a visita domiciliar ou outro mecanismo similar, caso a mãe se ausente das consultas ambulatoriais.

\section{Treinamento}

A Tabela 5 mostra o escore geral obtido relacionado ao número de profissionais treinados por hospital. Os hospitais com escore geral maior que $70 \%$ tiveram, em média, 12,5 profissionais treinados; os hospitais com escore geral entre 69 e $60 \%$ tiveram média de 13 profissionais; os entre 59 e $50 \%$ tiveram 6,6 profissionais em média; e, nos com escore abaixo de $50 \%$, a média foi de 5,0 profissionais treinados.
Tabela 3

Percentual médio geral de cumprimento das normas de implantação (E) segundo ser Hospital Amigo da Criança e contar com Banco de Leite Humano. São Paulo, Brasil, 2004.

\begin{tabular}{|c|c|c|c|c|c|c|}
\hline \multirow{3}{*}{$\begin{array}{l}\text { Hospital amigo } \\
\text { da criança }\end{array}$} & \multicolumn{4}{|c|}{ Banco de Leite Humano } & \multicolumn{2}{|c|}{ Total } \\
\hline & \multicolumn{2}{|c|}{ Sim } & \multicolumn{2}{|c|}{ Não } & \multirow[b]{2}{*}{$\mathrm{n}$} & \multirow[b]{2}{*}{$E(\%)$} \\
\hline & $\mathrm{n}$ & $E(\%)$ & $\mathrm{n}$ & $E(\%)$ & & \\
\hline Sim & 7 & 68,2 & 3 & 61,9 & 10 & 66,3 \\
\hline Não & 3 & 61,5 & 11 & 46,4 & 14 & 50,5 \\
\hline Total & 10 & 66,1 & 14 & 49,7 & 24 & 56,6 \\
\hline
\end{tabular}

Tabela 4

Percentual médio de cumprimento das normas de implantação por etapa implantada (E1, E2, E3), segundo o hospital ser Amigo da Criança (HAC) e contar com Banco de Leite Humano (BLH). São Paulo, Brasil, 2004.

\begin{tabular}{lccc}
\hline Hospitais & $\begin{array}{c}\text { 1a Etapa } \\
\text { E1 (\%) }\end{array}$ & $\begin{array}{c}\text { 2a Etapa } \\
\text { E2 (\%) }\end{array}$ & $\begin{array}{c}\text { 3a Etapa } \\
\text { E3 (\%) }\end{array}$ \\
\hline HAC & 79,4 & 65,5 & 50,0 \\
Com BLH & 76,9 & 65,3 & 50,0 \\
HAC+BLH & 80,3 & 65,7 & 50,0 \\
Não HAC+Sem BLH & 53,5 & 56,6 & 40,3 \\
\hline
\end{tabular}

\section{Indicadores periódicos}

Analisou-se a disponibilidades dos dados. Os resultados obtidos foram: mortalidade intrahospitalar dos RNBP - disponível em $60,7 \%$ dos hospitais; número de bebês que realizaram o Método Mãe Canguru - 46,4\%; número de bebês em Método Mãe Canguru reinternados - 50\%; tempo médio de permanência dos bebês em Método Mãe Canguru - 42,8\%; alimentação dos bebês em Método Mãe Canguru na alta $-46,4 \%$ e alimentação dos RNBP na alta $-22,4 \%$.

\section{Medidas humanizadoras}

\section{- Para os bebês}

A posição em flexão no "ninho" e o controle ambiental de luz são adotados pela maioria; o controle de som e respeito ao sono, em dois terços; e amenização dos procedimentos dolorosos, em um terço. 
Tabela 5

Escore geral de cumprimento das normas de implantação e número de profissionais treinados por hospital. São Paulo, Brasil, 2004.

\begin{tabular}{lc}
\hline Escore geral (E) & $\begin{array}{c}\text { Profissionais } \\
\text { treinados }(\mathrm{n})\end{array}$ \\
\hline 75,0 & 13 \\
72,6 & 15 \\
72,6 & 16 \\
71,4 & 13 \\
70,2 & 11 \\
70,2 & 9 \\
Escore acima de $70 \%$ & Média: 12,8 \\
69,0 & 20 \\
67,9 & 10 \\
65,5 & 10 \\
61,9 & 6 \\
60,7 & 19 \\
Escore entre 69 e $60 \%$ & Média: 13,0 \\
59,5 & 10 \\
58,3 & 5 \\
57,1 & 8 \\
57,1 & 5 \\
52,4 & 5 \\
Escore entre 59 e $50 \%$ & 5 \\
48,8 & 5 \\
47,6 & 5 \\
46,4 & 5 \\
45,2 & 5 \\
42,9 & 5,6 \\
9,5 & 5,0 \\
Escore abaixo de $50 \%$ & 5 \\
\hline
\end{tabular}

\section{- Para as mães}

Acesso irrestrito à UTI neonatal, acolhimento na primeira visita, participação nos cuidados e alimentação, poltronas confortáveis, refeições extras, outro local para relaxar, uma série de atividades de lazer, apoio psicológico, atividades corporais e ações educativas.

\section{- Para os pais}

Livre acesso à UTI neonatal, TV em alguns hospitais, refeições e auxílio transporte.

\section{- Para a família}

Permissão de visita ao bebê na UTI neonatal e permanência da avó na ausência da mãe, visita dos irmãos na UTI em alguns serviços, visita do pai e irmãos no alojamento canguru e, rara- mente, a participação de qualquer pessoa escolhida pela mãe para substituí-la.

\section{- Para os profissionais de saúde}

Dezessete equipes não recebem qualquer suporte, 11 delas são liberadas das atividades rotineiras para reuniões e cursos, duas equipes recebem apoio psicológico, e uma recebe auxílio financeiro para aprimoramento científico e pesquisa.

\section{Fatores motivadores}

Os principais motivos que desencadearam a implantação foram: humanização da assistência, fortalecimento do vínculo afetivo entre mães e bebês, melhoria dos cuidados como o RNBP e aumento das taxas de amamentação.

\section{Obstáculos}

O obstáculo encontrado em todos os hospitais foi a resistência dos profissionais às mudanças de rotinas. Também foram encontrados: a falta de leitos ou espaço físico inadequado para garantir o alojamento canguru e o ambulatório, sobrecarga de trabalho da equipe treinada para repassar o treinamento, dificuldades em captar mães, em manter uma relação multidisciplinar na equipe, em obter o compromisso familiar de retornar freqüentemente e a falta de recursos econômicos para reformas, alimentação e transporte aos familiares e para realizar visitas domiciliares.

\section{Estratégias de superação}

A resistência dos profissionais de saúde foi superada através de várias modalidades de treinamentos, ajuda de profissionais mais experientes, apoio da direção e caminhar lentamente. As dificuldades em captar mães foram superadas através de: flexibilização das regras, melhoria do conforto, facilitação do transporte, fornecimento de refeições, atividades de lazer, educativas, corporais, cursos e cordialidade da equipe. As dificuldades relativas a não contar com espaço físico adequado para alojamento canguru e/ou ambulatório foram superadas realizando o Método Mãe Canguru parcial, compartilhando salas originalmente com outras finalidades, usando o alojamento conjunto comum, atendendo ambulatorialmente os bebês na UTI neonatal (todas as acima acompanhadas de peso de alta nos critérios convencionais) e também desativação de outro setor ou reforma, com criação da unidade. 


\section{Obstáculos impeditivos}

Alguns obstáculos foram considerados intransponíveis levando ao abortamento da implantação do método ou de etapas: fechamento do setor de maternidade, mudança radical dos funcionários, falta de recursos financeiros, falta de apoio da direção em algumas situações decisivas, a resistência densa da equipe da UTI e as dificuldades de relacionamento entre os membros da equipe multidisciplinar.

\section{Discussão}

A coleta de dados através de um questionário enviado pelo correio tem limitações e pode conter desvios que teriam sido evitados se os dados fossem obtidos por meio de uma entrevista, acompanhada de observação local. Entrevistas com as mães também teriam proporcionado uma avaliação mais precisa do grau de implantação e das medidas humanizadoras. Entretanto, a dispersão dos hospitais pelo estado inviabilizou a realização do estudo nesses moldes.

\section{Grau de implantação}

Este estudo encontrou que ser HAC e contar com BLH foram fatores facilitadores da implantação. Segundo Carvalho \& Prochnik 18, o apoio ao aleitamento materno e o alojamento conjunto são condições fundamentais para efetivar as rotinas do Método Mãe Canguru. Nos hospitais credenciados na Iniciativa Hospital Amigo da Criança, as mães permanecem com seus bebês em alojamento conjunto, e as equipes são treinadas para manejar a lactação de RNBP 21. Essa iniciativa tem sido apontada como um programa de humanização da assistência na maternidade 22. Os BLH, além de pasteurizar, processar e disponibilizar leite materno para os bebês de risco, são considerados centros de orientação e suporte às mães com dificuldades em manter a lactação 23. Portanto, os hospitais com esses fatores já trilharam o caminho da humanização da assistência e sabem lidar com a amamentação de prematuros, o que explicaria esse achado.

Os hospitais escola foram os que conseguiram implantar melhor o programa, e os filantrópicos aqueles que tiveram mais dificuldades. Além do tipo de gerenciamento, parece ter influenciado essa diferença o fato de os hospitais escola concentrarem o maior número de HAC e com BLH, o contrário ocorrendo com os hospitais filantrópicos.
Os resultados referentes às etapas implantadas mostraram que a implantação do Método Mãe Canguru foi maior nas unidades neonatais, tanto em número de hospitais como em grau de implantação, seguida da implantação no alojamento canguru e, por último, no ambulatório de seguimento. Na primeira etapa, apesar do acesso liberado aos pais, as dificuldades estavam relacionadas com a captação e permanência da mãe na UTI neonatal, quando é exigida da equipe uma convivência mais estreita com ela. Esse obstáculo foi sendo superado à medida que os profissionais eram treinados e adquiriam experiência, e as condições oferecidas para a estadia da mãe melhoraram. Esses achados são compatíveis com as observações feitas por Kirsten et al. 24, quando descreveram que o sucesso da implantação depende, entre outros fatores, do treinamento dos profissionais de saúde e das facilidades e cortesias que o hospital oferece às mães para sua permanência.

Na segunda etapa, alojamento canguru, para as mães, as medidas humanizadoras foram variadas e em grande número, enquanto que aquelas voltadas para facilitar a presença do pai eram mais pobres e geralmente a família era excluída do programa, sendo raros os hospitais que facilitavam e incentivavam a sua presença. As dificuldades no cumprimento das normas de implantação encontradas na segunda etapa estavam relacionadas ao manejo da amamentação e à inclusão da família nas decisões de consenso com a equipe. Na terceira etapa, a fase ambulatorial, os hospitais apresentaram dificuldades para realizar visitas domiciliares, resultando na adoção de pesos mínimos para a alta próximos da atenção convencional. Esses resultados parecem indicar que a dificuldade de relacionamento entre a equipe e a família, que possibilitaria as tomadas de decisão em conjunto, gerou uma situação de insegurança quanto à continuidade da amamentação e dos cuidados no domicílio, possivelmente por desconhecimento do ambiente e da estrutura familiar, acabando por impedir a alta precoce. A importância da integração entre a equipe hospitalar e a família foi relatada por Toma 25, que defende que o sucesso do Método Mãe Canguru depende, além da vontade da mãe, do apoio de sua rede familiar e que a equipe, planejando em conjunto com ela, garanta que a criança receba os cuidados apropriados.

\section{Os profissionais de saúde}

A resistência dos profissionais às mudanças de rotinas, mesmo reconhecendo que o Método Mãe Canguru trazia melhorias para a assistên- 
cia ao RNBP, foi o obstáculo encontrado em todos os hospitais, e apenas duas instituições ofereciam alguma forma de apoio psicológico para a equipe. Esses achados são diferentes dos resultados de Lima et al. 26, que encontraram total aceitabilidade do Método Mãe Canguru pelos profissionais entrevistados no IMIP, um hospital escola terciário situado em Recife. Desde 1994, o IMIP não utilizava mais cuidados baseados em incubadoras, por considerálos inapropriados, muito caros, insuficientes e de difícil manutenção. Em comparação, todos os hospitais paulistas praticavam a assistência convencional ao RNBP concomitante ao Método Mãe Canguru, motivados pela humanização, melhoria do vínculo afetivo e da assistência, uma situação diferente do IMIP. A falta de recursos nem sempre facilita a adoção das novas rotinas. Lincetto et al. 27 , ao estudarem a implantação do Método Mãe Canguru em um hospital com recursos limitados, em Moçambique, encontraram uma grande resistência dos profissionais às mudanças, porque não estavam familiarizados com o Método Mãe Canguru e acreditavam que apenas melhores equipamentos poderiam aumentar a sobrevivência dos RNBP. O envolvimento progressivo dos profissionais e as discussões dos resultados obtidos foram gradativamente derrubando as resistências. Citam também que o apoio das autoridades de saúde do governo local e da direção do hospital foi fundamental nessa fase. Segundo Cecchetto 28, o foco de conflito da implantação dos programas de humanização perinatal, em hospitais com alta tecnologia, tem sido a luta entre um modelo médico de assistência, centralizador e paternalista, e a corrente humanista que acredita que o indivíduo doente, ou seus representantes, tem direito à informação e à participação na tomada das decisões durante o processo assistencial. Nogueira-Martins 29, em seus estudos sobre a humanização das relações assistenciais, refere que esse caminho é árduo, que as resistências não são pequenas e costumam ser crescentes no decorrer do processo. Também defende que o relacionamento interpessoal é um fator importante na facilitação da implantação desses programas e dá ênfase à criação de serviços de consultoria psiquiátrica e psicológica de apoio à tarefa assistencial, que ajudem os profissionais a se relacionarem com o cliente, com a família e entre si.

\section{O treinamento}

Este estudo encontrou que o treinamento tem uma grande importância na implantação. Vá- rias modalidades de treinamento foram desenvolvidas, a partir do oferecido pelo MS, para ajustar a implantação, e a perda ou desarticulação da equipe inicialmente treinada, responsável pelo repasse de informações, causou o abortamento parcial ou total do programa. Cattaneo et al. 5 e Kirsten et al. 24 enfatizam a importância do treinamento e concordam em que todos os profissionais da unidade neonatal devam ser capacitados, através de um treinamento de caráter multiprofissional, com conteúdo programático bastante amplo sobre os RNBP e o Método Mãe Canguru, que prepare a equipe para dar apoio psicoafetivo aos pais. Apesar de o curso ministerial obedecer a esses critérios, este estudo encontrou que o número facilitador da implantação situava-se em torno de 12 ou 13 profissionais por hospital, um número maior que os cinco definidos pelos critérios do programa e que, apesar do treinamento, a equipe resistia a delegar os cuidados dos prematuros aos pais.

\section{Os gestores}

A dificuldade em obter o apoio da direção para efetivar as mudanças na organização e na estrutura física dos hospitais, necessárias à implantação do método, também foi um dos resultados desse estudo. Ela foi responsável pelo abortamento do programa ou por adaptações que resultaram num Método Mãe Canguru parcial que levaram à adoção de pesos de alta próximos ao do cuidado convencional, prolongando o tempo de permanência da mãe. Conforme as estratégias para manter a mãe no hospital necessitaram ser mais variadas e criativas, envolvendo mais pessoas de diversos setores e levando a maiores mudanças na organização, a dificuldade em manter o apoio da direção foi crescendo e, em alguns casos, chegando ao esgotamento das iniciativas de implantação. Esses achados são compatíveis com as afirmações de Denis \& Champagne 30 que, analisando a implantação segundo uma perspectiva política, defendem que o sucesso de uma implantação depende de três fatores: de um suporte importante dado à intervenção pelos agentes de implantação, do exercício de um controle suficiente na organização para operacionalizar a intervenção e de uma forte coerência entre os motivos das modificações e os objetivos da intervenção.

\section{O plano de implantação}

Outros achados, como a falta de recursos financeiros para executar as reformas estrutu- 
rais e a alta freqüência de indisponibilidade dos indicadores periódicos, parecem indicar que o plano de implantação tem falhas e que seu controle não tem sido feito de maneira sistemática pela gerência dos hospitais ou pelas instituições governamentais. As normas de implantação não prevêem recursos orçamentários para adaptações do espaço físico, ou para a contratação dos profissionais especializados indicados para compor a equipe multidisciplinar, para a manutenção da internação da mãe por cinco dias mínimos após o parto, refeições e auxílio transporte, ou para as visitas domiciliares, mas orienta que o hospital obedeça a esses quesitos. Além disso, as normas estaduais de implantação, como as ministeriais, não prevêem recursos para o seguimento, monitorização e avaliação do programa. Furlan et al. 31 já levantaram essas contradições quando estudaram a percepção e vivência das mães que permaneciam no Método Mãe Canguru num hospital universitário de Ribeirão Preto, São Paulo.

\section{Conclusões e recomendações}

Este estudo permitiu concluir que a atenção humanizada ao RNBP - Método Mãe Canguru é um programa complexo de saúde e, como tal, tem inúmeros fatores que influenciam o processo de implantação e de avaliação.

Apesar de ser um programa ministerial, o foco da proposta de disseminação tem sido os profissionais de saúde, tornando-os agentes da implantação e deixando, à margem, os vários níveis de decisão do SUS.

Em São Paulo, a implantação do Método Mãe Canguru está em movimento, na direção da humanização. Nesse processo, a resistência dos profissionais à participação da família tem dado ao Método Mãe Canguru um caráter hospitalar, com o prolongamento das internações em alojamento conjunto e alta próxima aos critérios convencionais.
Ser hospital escola, ser HAC, contar com BLH e ter um número maior que 12 profissionais treinados foram identificadas como medidas facilitadoras da implantação do Método Mãe Canguru.

É recomendável que a capacitação tenha continuidade, com ajustes no treinamento para ampliar o número de profissionais treinados e as estratégias que incentivem a participação da família visando ao seu empoderamento nas decisões compartilhadas.

Os gerentes dos hospitais precisam ser incentivados a criar mecanismos de apoio aos profissionais de saúde para melhorar o relacionamento entre si, com as mães, pais e familiares. Também devem ser informados da necessidade de mudanças na organização a fim de facilitarem a entrada e estadia de familiares nas suas dependências.

Os hospitais precisam receber apoio durante a implantação. Para tanto, as normas que transformaram a técnica Mãe Canguru em política pública necessitam prever recursos orçamentários indispensáveis para a adoção, acompanhamento e avaliação do processo de implantação. Uma especial atenção deve ser dada aos hospitais com muitas dificuldades na implantação.

Os gestores estaduais, municipais e hospitalares precisam ser incluídos nas estratégias governamentais de disseminação e implantação do método.

A adoção da Iniciativa Hospital Amigo da Criança e dos BLH pelos hospitais deve ser incentivada pelos gestores governamentais, e as estratégias de sensibilização para o Método Mãe Canguru precisam ter continuidade para que mais hospitais venham a aderir ao método.

Mais estudos voltados para o processo de implantação são necessários para preencher as lacunas do conhecimento deixadas por este trabalho. 


\section{Resumo}

Este estudo foi elaborado para analisar a implantação do Método Mãe Canguru em 28 diferentes hospitais públicos do Estado de São Paulo, Brasil. Os hospitais escola, os amigos da criança, os com banco de leite humano e os com mais de 12 profissionais treinados obtiveram melhores escores de implantação. A resistência dos profissionais de saúde à participação da família tem dado um caráter intra-hospitalar ao método. Mudanças no treinamento, incluir os gestores no programa e prever recursos orçamentários para a adoção, avaliação e controle do Método Mãe Canguru são necessários.

Método Mãe Canguru; Prematuro; Política de Saúde

\section{Colaboradores}

A. J. Colameo escreveu o artigo, fez a revisão da literatura, captou e tabulou os dados, discutiu e analisou os resultados e elaborou as conclusões. M. F. Rea colaborou na elaboração do abstract do artigo, no desenho da pesquisa, na análise e discussão dos resultados.

\section{Agradecimentos}

A todos os hospitais que participaram do estudo pela dedicação e paciência.

\section{Referências}

1. Secretaria de Estado da Saúde de São Paulo. Boletim CIS/2000. N. 2. Mortalidade infantil. São Paulo: Secretaria de Estado da Saúde de São Paulo; 2000.

2. Lubchenko LO. Determinação do peso e idade gestacional. In: Avery G, organizador. Neonatologia. Rio de Janeiro: Médica e Científica; 1984. p. 207-27.

3. Lubchenko LO, Searls DT. Neonatal mortality rate: relationship to birth weight and gestational age. J Pediatr 1972; (84):4.

4. Ministério da Saúde. Manual de assistência ao recém-nascido. Brasília: Secretaria de Assistência à Saúde, Ministério da Saúde; 1994.

5. Cattaneo A, Davanzo R, Uxa F, Tamburlini G. Recommendations for the implementation of Kangaroo Mother Care for low birth weight infants. International Network on Kangaroo Mother Care. Acta Paediatr 1998; 87:440-5.

6. Organização Mundial da Saúde. Alimentação infantil - bases fisiológicas. São Paulo: Instituto de Saúde/IBFAN Brasil; 1994

7. Kennell JH, Klaus MH. Bonding: recent observations that alter perinatal care. Pediatr Rev 1998 9:4-12.

8. Lucas A, Morley R, Cole TJ, Lister G, LeesonPayne C. Breast milk and subsequent intelligence quotient in children born preterm. Lancet 1992; 339:261-4.

9. Bradley RH, Casey PH. Family environment and behavioral development of low-birth weight children. Dev Med Child Neurol 1992; 34:822-6.

10. Strathearn L, Gray PH, O'Callaghan MJ, Wood DO. Childhood neglect and cognitive development in extremely low birth weight infants: a prospective study. Pediatrics 2001; 108:142-51.

11. Symington A, Pinelli J. Developmental care for promoting development and preventing morbidity in preterm infants. (Cochrane Review) In: The Cochrane Library, Issue 1, 2004. Oxford: Update Software.

12. Furman L, Kennell J. Breast milk and skin-to-skin kangaroo care for premature infants. Avoiding bonding failure. Acta Paediatr 2000; 89:1280-3.

13. Charpak N, Figueroa Z, Hamel A. O método mãe canguru: pais e familiares dos bebês prematuros podem substituir as incubadoras. Rio de Janeiro: McGraw-Hill; 1999.

14. Anderson GC, Marks EA, Wahlberg V. Kangaroo care for premature infants. Am J Nurs 1986; 86: 807-9.

15. Colonna F, Uxa F, da Graca AM, de Vonderweld U. The "kangaroo-mother" method: evaluation of an alternative model for the care of low birth weight newborns in developing countries. Int J Gynaecol Obstet 1990; 31:355-9.

16. Ludington-Hoe SM, Anderson GC, Simpson S, Hollingsead A, Argote LA, Rey H. Birth-related fatigue in 34-36 week preterm neonates: rapid recovery with very early kangaroo (skin-to-skin) care. J Obstet Gynecol Neonatal Nurs 1999; 28:94-103.

17. Ludington-Hoe SM, Nguyen N, Swinth JY Satyshur RD. Kangaroo care compared to incubators in maintaining body warmth in preterm infants. Biol Res Nurs 2000; 2:60-73.

18. Carvalho MR, Prochnik M. Método Mãe-Canguru de atenção ao prematuro. http://federativo.bndes.gov.br/bf_bancos/experiencias/x0001959.pdf (acessado em Mai/2004).

19. Ministério da Saúde. Portaria GM n. 693 - Norma de orientação para a implantação do método canguru. Diário Oficial da União 2000; 5 jul.

20. Secretaria de Estado da Saúde de São Paulo. Resolução SS-62 de 5 de junho de 2001. Normas de atenção humanizada ao recém-nascido de baixo peso (método canguru) no Estado de São Paulo. Diário Oficial do Estado 2001; 6 jun.

21. Fundo das Nações Unidas para a Infância. Manejo e promoção do aleitamento materno: curso de 18 horas para equipes de maternidades. Brasília: Fundo das Nações Unidas para a Infância; 1993. 
22. Levin A. Humane neonatal care initiative. Acta Paediatr 1999; 88:353-5.

23. Secretaria de Estado da Saúde de São Paulo. Saúde no Estado de São Paulo: compromisso com o cidadão. São Paulo: Secretaria de Estado da Saúde de São Paulo; 2002.

24. Kirsten GF, Bergman NJ, Hann FM. Kangaroo mother care in the nursery. Pediatr Clin North Am 2001; 48:443-52.

25. Toma TS. Método Mãe Canguru: o papel dos serviços de saúde e das redes familiares no sucesso do programa. Cad Saúde Pública 2003; 19 Suppl 2:S233-42.

26. Lima G, Quintero-Romero S, Cattaneo A. Feasibility, acceptability and cost of kangaroo mother care in Recife, Brazil. Ann Trop Paediatr 2000; 20:22-6.

27. Lincetto O, Nazir AI, Cattaneo A. Kangaroo mother care with limited resources. J Trop Pediatr 2000; 46:293-5.
28. Cecchetto S. Mãe canguru: tecnologia perinatal humana. Parte I: uma abordagem pela bioética. In: Carvalho MR, Tamez RN, organizadores. Amamentação: bases científicas para a prática profissional. Rio de Janeiro: Guanabara Koogan; 2002. p. $132-6$.

29. Nogueira-Martins MCF. Humanização das relações assistenciais: a formação do profissional de saúde. São Paulo: Casa do Psicólogo; 2001.

30. Denis J-L, Champagne F. Análise da implantação. In: Hartz ZMA, organizadora. Avaliação em saúde: dos modelos conceituais à prática na análise de implantação de programas. Rio de Janeiro: Editora Fiocruz; 2000. p. 49-88.

31. Furlan CEFB, Scochi CGS, Furtado MCC. Percepção dos pais sobre a vivência no método mãecanguru. Rev Lat Am Enfermagem 2003; 11:44452 .

Recebido em 07/Dez/2004

Versão final reapresentada em 03/Jun/2005

Aprovado em 16/Set/2005 